\title{
Debris Rotation Analysis during Tethered Towing for Active Debris Removal
}

\author{
Pawel Jaworski*, Vaios Lappas ${ }^{\dagger}$, Antonios Tsourdos ${ }^{\star}$ and Iain Gray ${ }^{\S}$ \\ Cranfield University, Cranfield, Bedfordshire, MK43 OAL, United Kingdom \\ Hanspeter Schaub ${ }^{* *}$ \\ University of Colorado, Boulder, Colorado, 80309-0429, USA
}

\section{Nomenclature}

$$
\begin{aligned}
& \mathbf{A}=\text { rotation matrix } \\
& A=\text { tether cross-sectional area, } \mathrm{m}^{2} \\
& c \quad=\text { tether damping constant, } \mathrm{kg} / \mathrm{s} \\
& E \quad=\text { Young's modulus, } \mathrm{GPa} \\
& \hat{\boldsymbol{e}}=\text { tether link unit vector } \\
& \hat{\boldsymbol{e}}_{\Delta \theta}=\text { rotation axis unit vector } \\
& e \quad=\text { relative distance control error, } \mathrm{m} \\
& \boldsymbol{F}=\text { actuation force, } \mathrm{N} \\
& \mathbf{I}=\text { inertia tensor, } \mathrm{kg} \cdot \mathrm{m}^{2} \\
& \boldsymbol{K}_{I}=\text { tether heading integral gain matrix } \\
& \boldsymbol{K}=\text { tether heading proportional gain } \\
& k \quad=\quad \text { tether spring constant, } \mathrm{N} / \mathrm{m} \\
& k_{P}=\text { relative distance proportional gain } \\
& k_{D}=\text { relative distance derivative gain } \\
& k_{I}=\text { relative distance integral gain } \\
& \boldsymbol{L}=\text { external known torque, } \mathrm{Nm} \\
& \boldsymbol{l}=\text { tether link length vector, } \mathrm{m}
\end{aligned}
$$

\footnotetext{
*Postgraduate Student, School of Aerospace, Transport and Manufacturing.

${ }^{\dagger}$ Professor in Aerospace Systems, School of Aerospace, Transport and Manufacturing, vaios.lappas@cranfield.ac.uk, Senior Member AIAA.

"Director of Research, School of Aerospace, Transport and Manufacturing, a.tsourdos@cranfield.ac.uk, AIAA Member.

${ }^{\S}$ Director, School of Aerospace, Transport and Manufacturing.

*** Alfred T. and Betty E. Look Professor of Engineering, Aerospace Engineering Sciences Department, University of Colorado
} 


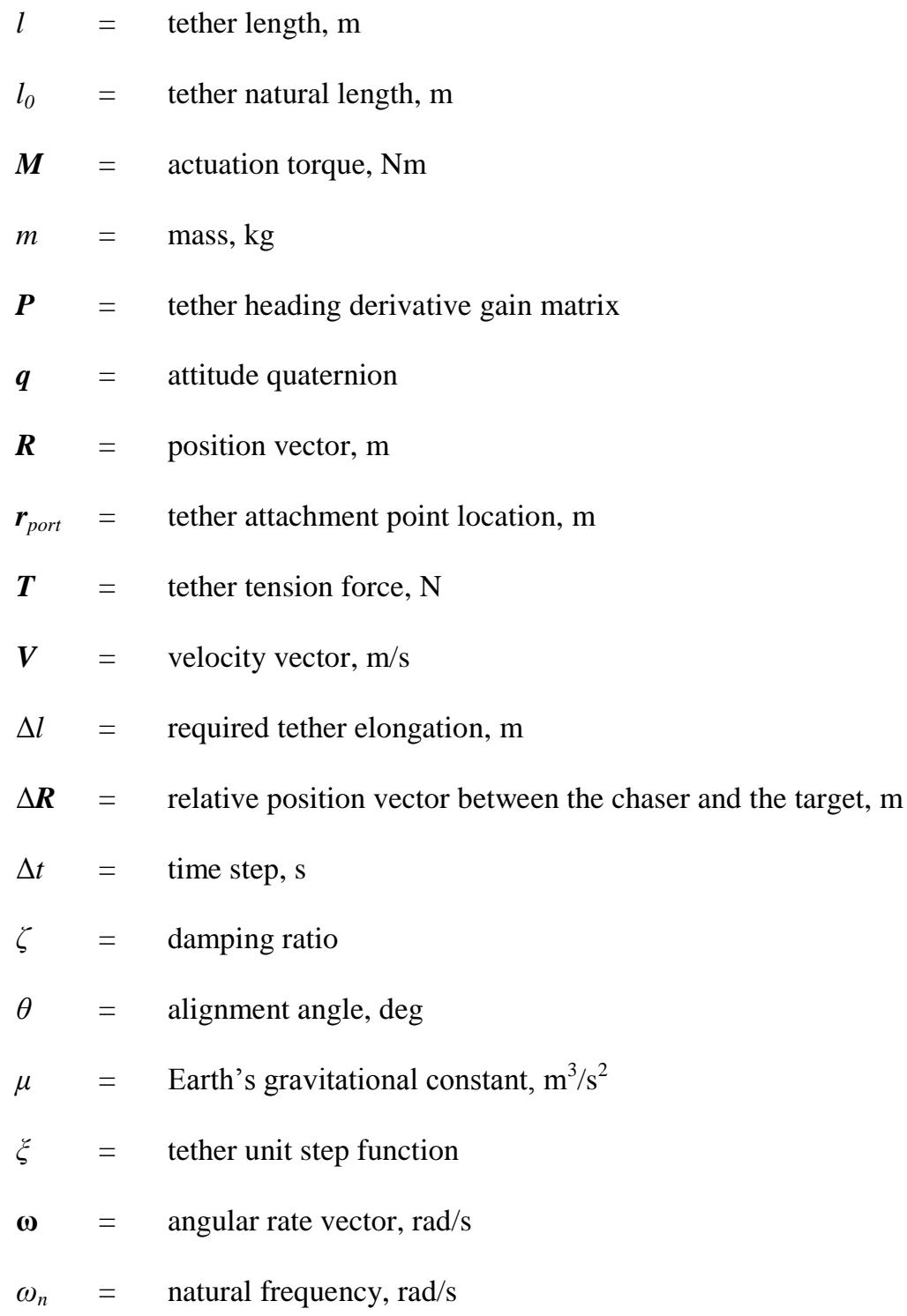




\section{Introduction}

TETHERED satellite systems (TSS) have been studied intensely in the last decades [1]. Dynamics of TSS have received increased attention in recent decades, due to their useful applications in space. Some of the TSS applications include orbital maneuvers, on-orbit servicing and studies using tethers for the upper parts of the atmosphere using deployed probes [1]. In recent years, the interest in TSS has increased even more, due to the growing number of space debris in orbit around the Earth. The majority of space debris occupies the Low Earth Orbit (LEO) environment, which poses a significant threat for on-orbit operations due to the risk of possible collisions. Studies show that the problem will continue to grow unless a number of inactive space debris are removed every year [2,3]. A typical mission scenario includes launching an active spacecraft which rendezvous with the inactive debris, captures the target (debris) and eventually deorbits both satellites via controlled re-entry in the Earth's atmosphere. Many concepts of ensuring the connection with the debris target have been investigated, including harpoons, nets, grapples and robotic arms [4-7]. The dynamics of the electrodynamic tethers and their interaction with the Earth's plasma and magnetic is the active field of research $[1,23]$. Towing the inactive target via a tether continuous to be a promising idea, and is being widely investigated [8-16].

Dynamics of a tethered systems with 2 large end bodies is challenging, with many aspects still unexplored. In a paper by Jasper and Schaub [10], the authors study the tether dynamics and continuous open-loop thrust input shaping to attenuate the violent dynamics of TSS and hence avoid the collision between the end bodies. This approach, however, can be challenging due to the discrete on/off thruster capabilities. Several discrete thrust input shaping techniques are studied in [11]. These approaches are more realistic for on-off thrusters and offer better performance with respect to a step input in terms of end bodies collision avoidance and target attitude motion. The target's angular rate during tethered towing is studied in [12], however, the target's attitude has not been analyzed in detail. Investigation of the target's attitude is important in an active debris removal (ADR) mission scenario in order to avoid a tether wrapping up around the target and thus avoiding possible in-orbit collisions. The authors in [10] also emphasized the influence of the tether parameters, such as length, Young's modulus and damping ratio, on the system dynamics. Aslanov and Yudintsev [14] analyze the rotational motion of the target, when constant low thrust is applied by the active spacecraft. The study reveals that initial target orientation or initial slack in the tether can lead to tether tangling around the target which can result in tether rupture thus creating new debris in orbit. The study, however, does not consider closed-loop control of the chaser and its attitude motion impact on the target 
rotation. Following a deorbit burn, closed loop control of the chaser's attitude and relative position with respect to the target is analyzed in [13]. Closed-loop control is advantageous due to the increased safety achieved by avoiding the collision between the end bodies and robustness for uncertain target mass and inertia properties. Nevertheless, closed-loop control adds complexity to the system, requires careful consideration of sensor performance and may increase the control effort due to sensor noise. In [23], Li, Zhu, et al., analyse the dynamics of the electrodynamic tethers and show that the thermal effects affect the stability of the current-conducting tethers. The authors in [25] address the challenges of accurate and robust time integration methods applied to the space tethers dynamic models.

This paper focuses on studying the attitude motion of the target for the critical parts of a tethered ADR mission, which are during and following a deorbit burn applied by an active chaser. A feedback control is developed to stabilize the tether and the target separation distance in a towing ADR mission. This improves safety substantially by reducing the danger of chaser-debris collision and thus fragmentation. The paper assumes that the challenging rendezvous and target capture has been already performed. The space debris is considered to be a passive, uncooperative satellite in low Earth orbit. The chaser is equipped with a large main engine $(\sim 2000 \mathrm{~N})$ for performing the deorbit burn and reaction control system (RCS) for applying force and torque corrections. The main engine is of an on-off type and the RCS is capable of delivering variable thrust. Following the deorbit burn, the chaser activates two closed-loop controllers. First, the relative distance between the end bodies is controlled to maintain a small tension in the tether, and second, the chaser's orientation is controlled to ensure the correct attitude of the chaser. The end bodies are connected by a discretized viscous-elastic tether. Based on the attitude motion analysis $[18,19]$ of the inactive Envisat satellite, which is the focus of the e.Deorbit mission [17], a representative ADR mission scenario is considered which accounts for small, residual initial angular rates of the target prior to the deorbit burn. Furthermore, no input shaping of the deorbit burn is used. The main thrust is modeled as a step function, which accounts for simplified and worst-case approach. However, the reader should note that a combination of discrete deorbit burn shaping and closed-loop control following the deorbit burn may improve the performance. In the proposed analysis, the emphasis is placed on avoiding the tether tangling around the target which can result in tether rupture or debris collision and thus debris fragmentation.

The goal of this study is to illustrate the impact of the tether parameters on the attitude motion of a passive target and to determine the desirable tether parameters, which combined with design of a closed-loop controller, can ensure damping of a target's rotation motion in a realistic ADR mission scenario. 
This paper is organized as follows. In Section II, the equations of motion for the six degrees of freedom end bodies connected by a discretized viscous-elastic tether are developed. Section III gives an overview of the relative distance and chaser's attitude controllers. Because the performance of the closed-loop controllers is not the major scope of the paper, simple PID controllers have been developed. Section IV discusses the numerical simulation results and finally section $\mathrm{V}$ draws the conclusions.

\section{Equations of Motion}

The dynamics system considered in the paper consists of a chaser, debris and a tether connecting the two bodies, see Fig. 1. Each of the end bodies is modeled as a rigid body which can translate and rotate, resulting in a 6 degrees of freedom model. The target is assumed to be a passive, inactive satellite. The chaser has an active control system which is able to deliver both pure force and torque. The tether is discretized into 2 point masses and 3 equidistant massless tether links. Each tether element is modeled as a parallel spring-damper system. When a given tether link length, $l_{i}, i=\{1,2,3\}$, is smaller than its natural length, $l_{0, i}$, the tether force in a given tether element vanishes. Discretized tether model has been widely used in the previous studies [9-13]. It accounts for transverse oscillations of the tether and adds only little complexity into the system. The effect of the number of the tether nodes on the behavior of the system has been studied in $[12,13]$. The number of the tether nodes greater than 2 does not change considerably the observed behavior of the system, but adds significant computation time. For these reasons, the tether has been discretized into 2 point masses.

It is assumed that the tether attachment points on the chaser and target are not located in the center of mass of the end bodies, but are displaced relatively to the center of mass of each body. The displacement of the tether

attachment points with respect to the center of mass is described by the vectors $\boldsymbol{r}_{p o r t, C}$ and $\boldsymbol{r}_{p o r t, T}$, for the chaser and the target, respectively. Therefore, when the neighboring tether links connected to the end bodies are stretched, the torque is applied. It must be emphasized, that when the deployed net is considered, there will be many attachment points between the net and the target. Due to numerous contact points, the damping of the target rotational motion will likely increase [13]. In this paper, one tether attachment point is considered which considerably simplifies the model and can be regarded as a worst-case scenario in terms of target angular rate damping. 


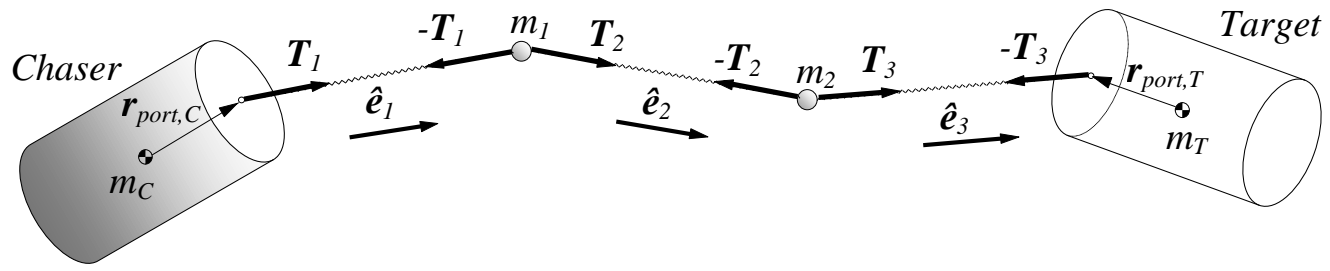

Fig. 1 Discretized tether model with 2 point masses and 3 tether elements

\section{A. End Bodies Dynamics}

Translational and rotational motion of each end body is fully described by its inertial position, $\boldsymbol{R}$, and inertial velocity, $\boldsymbol{V}$, in the Earth-Centered Inertial (ECI) frame. The ECI frame has its origin in the center of mass of the Earth, the $\mathrm{Z}$ axis points towards the North Pole, the $\mathrm{X}$ axis is aligned with the vernal equinox and the $\mathrm{Y}$ axis completes the right-handed orthogonal frame and lies in the equatorial plane. Rotational motion of each end body is described by attitude quaternion, $\boldsymbol{q}$, and angular rate vector in the body frame, $\boldsymbol{\omega}$. The attitude quaternion of each body is expressed as $\boldsymbol{q}=\left[\begin{array}{llll}q_{1} & q_{2} & q_{3} & q_{4}\end{array}\right]^{T}$, where $q_{4}$ denotes the quaternion scalar part. The attitude quaternion, $\boldsymbol{q}$, describes the satellite orientation with respect to the ECI frame. The angular rate vector of each body with respect to the ECI frame is expressed in a given body frame as $\boldsymbol{\omega}=\left[\begin{array}{lll}p & q & r\end{array}\right]^{T}$.

The kinematic relationships for each end body are given by:

$$
\begin{gathered}
\dot{\boldsymbol{R}}=\boldsymbol{V} \\
\dot{\boldsymbol{q}}=\frac{1}{2}\left[\begin{array}{ccc}
q_{4} & -q_{3} & q_{2} \\
q_{3} & q_{4} & -q_{1} \\
-q_{2} & q_{1} & q_{4} \\
-q_{1} & -q_{2} & -q_{3}
\end{array}\right]\left[\begin{array}{l}
p \\
q \\
r
\end{array}\right]
\end{gathered}
$$

The translational inertial acceleration of each end body is given by:

$$
m \ddot{\boldsymbol{R}}=-m \mu \frac{\boldsymbol{R}}{|\boldsymbol{R}|^{3}}+\boldsymbol{F}+\xi \boldsymbol{T}
$$

where all vectors are expressed in inertial frame. The first term on the right hand side of Eq. (3) denotes the Earth gravitational force, $\boldsymbol{F}$ corresponds to the actuation force, $\boldsymbol{T}$ is a force applied by the tether link neighboring a given end body, and $\xi$ is a unit step function, defined for each tether element as: 


$$
\xi= \begin{cases}1, & l-l_{0}>0 \\ 0, & l-l_{0} \leq 0\end{cases}
$$

Note that for the passive target, actuation force equals $0, \boldsymbol{F}=0$.

The attitude dynamics of each end body is given by the Euler equation:

$$
\dot{\boldsymbol{\omega}}=\mathbf{I}^{-1}\left(\boldsymbol{M}+\boldsymbol{M}_{g g}+\boldsymbol{r}_{p o r t} \times \xi \boldsymbol{T}-\boldsymbol{\omega} \times \mathbf{I} \boldsymbol{\omega}\right)
$$

where all vectors are expressed in body frame. The first term in the parenthesis on the right hand side of Eq. (5), $\boldsymbol{M}$, denotes the actuation torque, $\boldsymbol{M}_{g g}$ corresponds to the gravity gradient torque, third term denotes the torque generated by the neighboring tether link force and the last term is the gyroscopic term. Note, that for the passive target, the actuation torque is $\boldsymbol{M}=0$.

\section{B. Tether Dynamics}

The tether is discretized into 2 point masses. The position of the masses in the ECI frame is denoted by $\boldsymbol{R}_{1}$ and $\boldsymbol{R}_{2}$. The velocities of the tether point masses in ECI frame are simply given by:

$$
\dot{\boldsymbol{R}}_{1}=\boldsymbol{V}_{1}, \quad \dot{\boldsymbol{R}}_{2}=\boldsymbol{V}_{2}
$$

For each tether element, the tether tension force is directed along the line of each link, which can be described by the unit vectors, defined as:

$$
\begin{gathered}
\hat{\boldsymbol{e}}_{1}=\frac{\boldsymbol{l}_{1}}{\left|\boldsymbol{l}_{1}\right|}=\frac{\boldsymbol{R}_{1}-\boldsymbol{R}_{C}-\mathbf{A}_{C}^{I} \boldsymbol{r}_{p o r t, C}}{\left|\boldsymbol{R}_{1}-\boldsymbol{R}_{C}-\mathbf{A}_{C}^{I} \boldsymbol{r}_{p o r t, C}\right|} \\
\hat{\boldsymbol{e}}_{2}=\frac{\boldsymbol{l}_{2}}{\left|\boldsymbol{l}_{2}\right|}=\frac{\boldsymbol{R}_{2}-\boldsymbol{R}_{1}}{\left|\boldsymbol{R}_{2}-\boldsymbol{R}_{1}\right|} \\
\hat{\boldsymbol{e}}_{3}=\frac{\boldsymbol{l}_{3}}{\left|\boldsymbol{l}_{3}\right|}=\frac{\boldsymbol{R}_{T}+\mathbf{A}_{T}^{I} \boldsymbol{r}_{p o r t, T}-\boldsymbol{R}_{2}}{\left|\boldsymbol{R}_{T}+\mathbf{A}_{T}^{I} \boldsymbol{r}_{p o r t, T}-\boldsymbol{R}_{2}\right|}
\end{gathered}
$$

where the subscripts ' $C$ ' and ' $T$ ' correspond to the chaser and the target, respectively. For convenience, length vectors corresponding to each tether links are defined, $\boldsymbol{l}_{i}$ for $i=\{1,2,3\}$. The magnitude of the vector $\boldsymbol{l}_{i}$ equals the length of a given tether link and the direction corresponds to the direction of each tether link. The tether attachment vectors, $\boldsymbol{r}_{p o r t, C}$ and $\boldsymbol{r}_{p o r t, T}$, are expressed in body frames and the position vectors $\boldsymbol{R}_{C}, \boldsymbol{R}_{T}, \boldsymbol{R}_{1}, \boldsymbol{R}_{2}$ are expressed in the 
ECI frame. Therefore, the tether attachment vectors have to be transformed to the ECI frame, hence the attitude matrices $\mathbf{A}_{C}^{I}$ and $\mathbf{A}_{T}^{I}$ are used.

The magnitude of the tether tension in each link can be calculated using simple one-dimensional model. Total tether tension in each link, $\boldsymbol{T}_{i}$, consists of two elements: an elastic term, $\boldsymbol{T}_{e}$, and a viscous term, $\boldsymbol{T}_{v}$, which are given by:

$$
\begin{gathered}
\boldsymbol{T}_{e, i}=\xi_{i} k\left(\left|\boldsymbol{l}_{i}\right|-l_{0, i}\right) \hat{\boldsymbol{e}}_{i} \\
\boldsymbol{T}_{v, i}=\left(\xi_{i} c \frac{\mathrm{d}}{\mathrm{d} t}\left|\boldsymbol{l}_{i}\right|\right) \hat{\boldsymbol{e}}_{i}
\end{gathered}
$$

where $\xi_{i}$ corresponds to the unit step function defined in Eq. (4) for each tether element, $c$ is a damping constant for each tether link and $k$ denotes the tether spring constant:

$$
k=\frac{E A}{l_{0, i}}
$$

where $E$ corresponds to the Young modulus of the tether material, $A$ is a cross-section area of the tether, and $l_{0, i}$ is the natural length of each tether link. Note that the sign convention in Eq. (8) is defined according to the sign convention of the unit vectors in Eq. (7), see also Fig. 1.

Time derivative of the magnitude of the tether link length vector, $\boldsymbol{l}_{i}$ in Eq. (8.b), can be calculated by projecting the relative velocities of the adjacent tether nodes on the tether element's line. Using this approach, the time derivatives of the tether link lengths are given by:

$$
\begin{gathered}
\frac{\mathrm{d}}{\mathrm{d} t}\left|\boldsymbol{l}_{1}\right|=\left(\boldsymbol{V}_{1}-\boldsymbol{V}_{p o r t, C}\right)^{T} \hat{\boldsymbol{e}}_{1} \\
\frac{\mathrm{d}}{\mathrm{d} t}\left|\boldsymbol{l}_{2}\right|=\left(\boldsymbol{V}_{2}-\boldsymbol{V}_{1}\right)^{T} \hat{\boldsymbol{e}}_{2} \\
\frac{\mathrm{d}}{\mathrm{d} t}\left|\boldsymbol{l}_{3}\right|=\left(\boldsymbol{V}_{p o r t, T}-\boldsymbol{V}_{2}\right)^{T} \hat{\boldsymbol{e}}_{3}
\end{gathered}
$$

For the first and third tether links, the inertial velocity of the tether attachment points in the ECI frame is obtained according to the following equations: 


$$
\begin{aligned}
& \boldsymbol{V}_{\text {port }, C}=\boldsymbol{V}_{C}+\mathbf{A}_{C}^{I}\left(\boldsymbol{\omega}_{C} \times \boldsymbol{r}_{\text {port }, C}\right) \\
& \boldsymbol{V}_{\text {port }, T}=\boldsymbol{V}_{T}+\mathbf{A}_{T}^{I}\left(\boldsymbol{\omega}_{T} \times \boldsymbol{r}_{\text {port }, T}\right)
\end{aligned}
$$

Finally, tether nodes translational dynamics equations are given by:

$$
m_{i} \ddot{\boldsymbol{R}}_{i}=-m_{i} \mu \frac{\boldsymbol{R}_{i}}{\left|\boldsymbol{R}_{i}\right|^{3}}-\xi_{i} \boldsymbol{T}_{i}+\xi_{i+1} \boldsymbol{T}_{i+1}
$$

\section{Closed-Loop Control}

The chaser has an active guidance and control system which allows it to control its position and attitude. During the deorbit burn, the chaser performs the attitude control required to align the chaser in its Local-Vertical/LocalHorizontal (LVLH) frame, to ensure the thrust is applied in correct direction. The LVLH frame's origin is located in the spacecraft's center of mass. The Z axis is aligned with the local nadir vector and points towards the Earth's center of mass, the $\mathrm{Y}$ axis is perpendicular to the orbital plane and points in the opposite direction to the orbital angular velocity. The $\mathrm{X}$ axis completes the right-handed orthogonal frame. To control the chaser's attitude during the deorbits burn, the attitude PD controller from [22] has been used. The detailed analysis of the attitude controller during the deorbit burn is not the focus of this study, and will not be studied in subsequent sections. Its performance is satisfactory, achieving zero Euler angle orientation in the chaser's LVLH frame with a $0.1^{\circ}$ accuracy. Three Euler angles $(\psi, \vartheta, \phi)$ denoted by yaw, pitch and roll, represent the attitude expressed in terms of 3 subsequent rotations performed around $\mathrm{Z}, \mathrm{Y}$ and $\mathrm{X}$ axes, respectively. The relationship between the quaternion and Euler angle representation can be given in terms of the attitude matrix transforming the vector from the reference frame to the body frame, so that $\mathbf{A}(\mathbf{q})=\mathbf{A}(\psi, \vartheta, \phi)$ :

$$
\begin{aligned}
\mathbf{A}(\mathbf{q}) & =\left[\begin{array}{ccc}
q_{1}^{2}-q_{2}^{2}-q_{3}^{2}+q_{4}^{2} & 2\left(q_{1} q_{2}+q_{3} q_{4}\right) & 2\left(q_{1} q_{3}-q_{2} q_{4}\right) \\
2\left(q_{2} q_{1}-q_{3} q_{4}\right) & -q_{1}^{2}+q_{2}^{2}-q_{3}^{2}+q_{4}^{2} & 2\left(q_{2} q_{3}+q_{1} q_{4}\right) \\
2\left(q_{3} q_{1}+q_{2} q_{4}\right) & 2\left(q_{3} q_{2}-q_{1} q_{4}\right) & -q_{1}^{2}-q_{2}^{2}+q_{3}^{2}+q_{4}^{2}
\end{array}\right] \\
\mathbf{A}(\psi, \vartheta, \phi) & =\left[\begin{array}{ccc}
c \vartheta c \psi & c \vartheta s \psi & -s \vartheta \\
-c \phi s \psi+s \phi s \vartheta c \psi & c \phi c \psi+s \phi s \vartheta s \psi & s \phi c \vartheta \\
s \phi s \psi+c \phi s \vartheta c \psi & -s \phi c \psi+c \phi s \vartheta s \psi & c \phi c \vartheta
\end{array}\right]
\end{aligned}
$$

Following the deorbit burn, the chaser controls the separation distance with respect to the target and the chaser's attitude. Two feedback controllers are used: a linear separation distance PID controller and a linear tether heading 
controller. The closed-loop control is a means to reduce the risk of the tether tangling around the target and it increases the robustness for initial target angular rates. Relatively simple guidance and control schemes are considered in this work and detailed analysis of their performance is beyond the scope of this paper.

Our study does not include navigation or sensor noise concerns. Furthermore, this paper considers the continuous force and torque variations to be perfectly implemented. This means that no thrusters dynamics are included. When a higher fidelity mission scenario is considered, control implementation limitations such as pulse width modulation and minimum impulse bit need to be addressed. For the scope of this paper such noise inclusions and thrusters dynamics would mask some subtleties of the dynamics being studied. Sensing the relative motion, and the impact of the sensor noise on the closed loop performance is beyond the scope of this note.

\section{A. Relative Distance PID Controller}

In previous studies $[9-10,15,16]$, it is shown that the slack in the tether should be avoided for safety considerations. When the tether is slack, the target is allowed to move freely, which can be dangerous for numerous reasons. First, slack in the tether results in more violent dynamics and reduces the closest approach of the end bodies which increases the probability of collision and thus fragmentation. Secondly, free rotation of the target can lead to tether tangling and eventually its rupture. For those reasons, the relative distance control law is developed which maintains a small residual tension in the tether.

The relative distance error, $e$, is given by:

$$
e=l_{0}+\Delta l+\left|\boldsymbol{r}_{p o r t, C}\right|+\left|\boldsymbol{r}_{p o r t, T}\right|-|\Delta \boldsymbol{R}|
$$

where $l_{0}$ is the natural length of the tether, $\Delta l$ is a design parameter corresponding to the desired tether elongation, and $\Delta \boldsymbol{R}=\boldsymbol{R}_{C}-\boldsymbol{R}_{T}$ is a distance between the chaser and the target centers of mass. The form of Eq. (13) accounts for the desired one-dimensional configuration of the system, when the vectors $\boldsymbol{r}_{p o r t, C}$ and $\boldsymbol{r}_{p o r t, T}$ are aligned with the tether, see Fig. 2. Note that the control approach is simplified, since it does not require accurate current tether length computation. Instead, the error in Eq. (13) is formed using the relative distance between the chaser and the target, $|\Delta \boldsymbol{R}|$, and desired final one-dimensional configuration. 


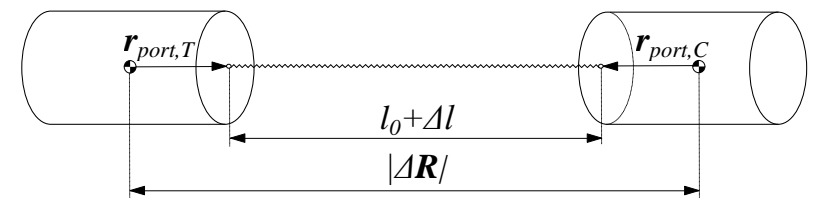

Fig. 2 Desired configuration of the system when the tether is taut

Differentiating Eq. (13) with respect to time yields:

$$
\dot{e}=-\left(\boldsymbol{V}_{C}-\boldsymbol{V}_{T}\right)^{T} \frac{\Delta \boldsymbol{R}}{|\Delta \boldsymbol{R}|}
$$

Equation (14) is obtained by projecting relative velocity between the chaser and target on the relative distance vector between the end bodies centers of mass.

Next, the commanded magnitude of the force is given by a simple proportional, derivative and integral control law:

$$
F_{\text {comm }}=k_{P} e+k_{D} \dot{e}+k_{I} \int_{0}^{t} e \mathrm{~d} t
$$

The integral term in Eq. (15) is approximated using the rectangle method:

$$
\int_{0}^{t} e \mathrm{~d} t \approx \sum_{n=0}^{t} e_{n} \Delta t
$$

Finally, the commanded thrust obtained in Eq. (15) is applied in the direction of the relative distance between the chaser and the target, which gives:

$$
\boldsymbol{F}_{\text {comm }}=F_{\text {comm }} \frac{\Delta \boldsymbol{R}}{|\Delta \boldsymbol{R}|}
$$

\section{B. Tether Heading Feedback Control}

Following the deorbit burn, the attitude of the chaser is controlled, so that it is aligned with the tether. For both satellites, the alignment angle, $\theta$, is defined as the angle between the tether attachment point vector and the tether line, see Fig. 3. Thus, for the chaser, the desired attitude is determined by the vectors $\boldsymbol{r}_{\text {port, }}$ and $-\Delta \boldsymbol{R}$ being parallel, and hence the desired chaser's alignment angle equal to null. This configuration ensures that the tether does not apply any torque to the chaser. 


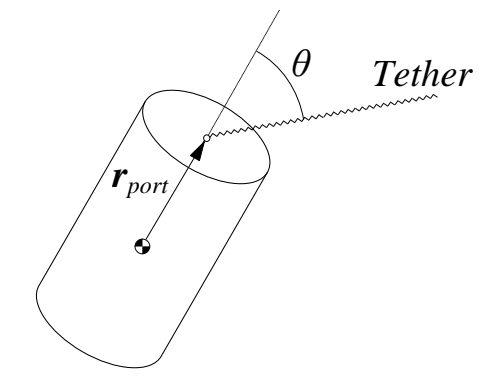

Fig. 3 Alignment angle $\theta$ definition

The attitude quaternion error, $\boldsymbol{q}_{e}$, is defined as:

$$
\boldsymbol{q}_{e}=\left[\begin{array}{c}
\hat{\boldsymbol{e}}_{\Delta \theta} \sin \frac{\theta_{C}}{2} \\
\cos \frac{\theta_{C}}{2}
\end{array}\right]
$$

where $\hat{\boldsymbol{e}}_{\Delta \theta}$ is a unit vector denoting the rotation axis around which the chaser must be rotated to make vectors $\boldsymbol{r}_{\text {port }, C}$ and $-\Delta \boldsymbol{R}$ parallel, and $\theta_{C}$ is the chaser's alignment angle, defined as:

$$
\hat{\boldsymbol{e}}_{\Delta \theta}=\frac{\Delta \boldsymbol{R} \times \boldsymbol{r}_{p o r t, C}}{\left|\Delta \boldsymbol{R} \times \boldsymbol{r}_{p o r t, C}\right|}, \quad \theta_{C}=\operatorname{acos}\left(\frac{-\Delta \boldsymbol{R}^{T} \boldsymbol{r}_{p o r t, C}}{|\Delta \boldsymbol{R}| \cdot\left|\boldsymbol{r}_{p o r t, C}\right|}\right)
$$

Since the chaser's attitude is controlled relatively to the tether, the chaser will track the direction of the tether. When this approach is combined with the relative distance control, it is evident that no global position and attitude control of the chaser is performed. The whole system will tend to oscillate or tumble around the nadir direction, achieving stable gravity gradient configuration [10]. Since the orientation of the whole system varies very slowly in time, the desired angular rate of the chaser is set to around all body axes as $\boldsymbol{\omega}_{\text {ref }}=0$.

The Lyapunov attitude control law from [21] has been simplified to the simple PID control law, neglecting the gyroscopic term and external, known torque. The known external torque can be approximated by a torque applied by a tether, which would require accurate computation of the tether force. Nevertheless, since the angle between the vectors $\boldsymbol{r}_{p o r t, C}$ and $-\Delta \boldsymbol{R}$ is very small, the torque $\boldsymbol{L}$ can be neglected. On the other hand, the gyroscopic term is proportional to the square of the chaser's angular rate which is approximately equal to the orbital rate, hence the gyroscopic term is negligible as well. This gives the simplified attitude PID control law: 


$$
\begin{gathered}
\boldsymbol{T}_{\text {comm }}=\boldsymbol{K} \boldsymbol{q}_{e, 1: 3} \operatorname{sign}\left(q_{e, 4}\right)-\tilde{\boldsymbol{P}} \boldsymbol{\omega}_{C}+\tilde{\boldsymbol{K}}_{I} \int_{0}^{t} \boldsymbol{q}_{e, 1: 3} \operatorname{sign}\left(q_{e, 4}\right) \mathrm{d} t \\
\tilde{\boldsymbol{P}}=\boldsymbol{P}+\boldsymbol{P} \boldsymbol{K}_{I} \mathbf{I}, \quad \tilde{\boldsymbol{K}}_{I}=\boldsymbol{K} \boldsymbol{P} \boldsymbol{K}_{I}
\end{gathered}
$$

where $\operatorname{sign}(\cdot)$ is the signum function which ensures the shortest distance to the reference attitude.

\section{Simulation Results}

\section{A. ADR Mission Scenario Overview}

The analyzed ADR scenario begins with the target and the chaser in a circular orbit with an altitude of $h_{0}=800$ $\mathrm{km}$. It is assumed that the chaser has performed successful rendezvous and target capture. The chaser is located behind the target and along the orbital in-track direction. The tether is initially taut, with a residual elongation equal to $5 \mathrm{~mm}$. Initial Euler angles of the chaser and target in their respective LVLH frames are zero. Initially, the chaser does not rotate in its LVLH frame, hence its angular rate equals the orbital rate. The target, however, has non-zero initial angular rate in its LVLH frame. According to [14], the dynamics of the system does not differ significantly with the direction of the target initial angular momentum vector. Thus, target initial angular rate in the body axes is set arbitrarily to:

$$
\boldsymbol{\omega}_{T, 0}=[3.5,-3,3]^{T} \mathrm{deg} / \mathrm{s},
$$

which corresponds to the magnitude of $\left|\boldsymbol{\omega}_{T, 0}\right|=5.5 \mathrm{deg} / \mathrm{s}$.

At $t=0$, the chaser initiates a $2000 \mathrm{~N}$ deorbit burn along the negative $\mathrm{X}$ axis direction in the chaser's LVLH frame. The deorbits burn is simulated as a step function with a duration of $200 \mathrm{~s}$. During this phase, the chaser controls its attitude using the PD controller. Following the deorbit burn, the chaser activates the closed-loop controllers described in Sec. III, to keep the separation distance between the end bodies constant and control the chaser's attitude.

\section{B. Simulation Parameters}

The chaser's and target's masses are kept constant for the mission duration and equal $2500 \mathrm{~kg}$ and $1500 \mathrm{~kg}$, respectively. The end bodies are modeled as perfect cylinders, which yields the diagonal inertia tensors given by: 


$$
\begin{aligned}
& \mathbf{I}_{C}=\operatorname{diag}[3000,10000,10000] \mathrm{kg} \cdot \mathrm{m}^{2} \\
& \mathbf{I}_{T}=\operatorname{diag}[1000,7000,7000] \mathrm{kg} \cdot \mathrm{m}^{2}
\end{aligned}
$$

It is assumed that the tether attachment points are displaced by half of the cylinder height along the respective body $\mathrm{X}$ axis, which gives:

$$
\begin{aligned}
\boldsymbol{r}_{p o r t, C} & =[3.2,0,0]^{T} \mathrm{~m} \\
\boldsymbol{r}_{p o r t, T} & =[-3.6,0,0]^{T} \mathrm{~m}
\end{aligned}
$$

For the given satellite masses, thrust level, $T$, and thrust duration, $\Delta \tau$, the chaser applies a velocity change equal to $100 \mathrm{~m} / \mathrm{s}$ which is obtained using the following equation:

$$
\Delta v=\frac{T \cdot \Delta \tau}{m_{C}+m_{T}}
$$

The velocity change of $100 \mathrm{~m} / \mathrm{s}$ corresponds to reducing the orbit's perigee to $h_{p}=428 \mathrm{~km}$. This can be calculated using the obit's energy equation:

$$
\frac{\left(v_{0}-\Delta v\right)^{2}}{2}-\frac{\mu}{R_{E}+h_{0}}=-\frac{\mu}{2 a}=-\frac{\mu}{2 R_{E}+h_{0}+h_{p}}
$$

where $R_{E}$ is the Earth's radius equal to $6371 \mathrm{~km}, a$ denotes the orbit's semimajor axis after the de-orbit burn has been applied and $v_{0}$ is the orbital velocity at the initial circular orbit, given by:

$$
v_{0}=\sqrt{\frac{\mu}{R_{E}+h_{0}}}
$$

In this analysis, the aerodynamic forces are omitted for simplicity. However, below the altitude of $500 \mathrm{~km}$, the detailed analysis should be performed, since the differential aerodynamic forces can lead to the end bodies tumbling and the whole tethered system's oscillations [23].

Six tether models with different characteristics are selected for detailed investigation. Since this paper focuses on the analysis of the attitude motion of the target, the tether length is not varied and a length $1 \mathrm{~km}$ in each case is used. Three values of Young's modulus, $E$, are selected corresponding to Nylon, Technora and Kevlar and two levels of 
damping ratios, $c$, are used. The parameters are based on the tether characteristics proposed by the ESA for further investigation [24]. The tether parameters used in the analysis are presented in Table 1.

Table 1 Tether parameters in the analysis

\begin{tabular}{lccccccc}
\hline \hline Model & $E, \mathrm{GPa}$ & $A, \mathrm{~mm}^{2}$ & $l_{0}, \mathrm{~m}$ & $m, \mathrm{~kg}$ & $c, \mathrm{~kg} / \mathrm{s}$ & $k, \mathrm{~N} / \mathrm{m}$ & $\Delta l, \mathrm{~m}$ \\
\hline Nylon-LD & 1 & 8 & 1000 & 9.2 & 0.001 & 8 & 0.5 \\
Technora-LD & 50 & 8 & 1000 & 11.2 & 0.001 & 402 & 0.01 \\
Kevlar-LD & 170 & 8 & 1000 & 11.6 & 0.001 & 1367 & 0.003 \\
Nylon-HD & 1 & 8 & 1000 & 9.2 & 0.3 & 8 & 0.5 \\
Technora-HD & 50 & 8 & 1000 & 11.2 & 0.3 & 402 & 0.01 \\
Kevlar-HD & 170 & 8 & 1000 & 11.6 & 0.3 & 1367 & 0.003 \\
\hline \hline
\end{tabular}

The last column in Table 1 gives the desired tether elongation - relative separation distance control error, as given by Eq. (13). The values are chosen so that each case results in the desired tether tension arbitrarily set to $4 \mathrm{~N}$. Considering the chaser and target masses, it requires $10.7 \mathrm{~N}$ of thrust to be delivered continuously at the desired configuration. The feasibility of $\Delta l$ is not considered in this paper.

The spring constant, $k$, and damping constant, $c$, in Table 1 is given for the full tether. To obtain the spring constant for each tether link, the spring constant given in Table 1 must be multiplied by the number of tether elements, thus in our case by 3 . This also refers to the damping constant for each tether link [13].

Numerical integration is performed using the Matlab's Runge-Kutta ode45 function with a relative tolerance equal to $10^{-3}$. The control update frequency is set to $10 \mathrm{~Hz}$ and during the time step of $\Delta t=0.1 \mathrm{~s}$, the chaser applies constant commanded force and torque.

\section{Closed-loop Gains}

Proportional and derivative gains in the relative distance controller in Eq. (15), are obtained on the basis of an one-dimensional massless spring-damper model with 2-point end masses, corresponding to the debris and the chaser. A natural frequency and a damping ratio of the linear closed-loop system are given by:

$$
\omega_{n}=\sqrt{\frac{E A}{l_{0}}\left(\frac{1}{m_{T}}+\frac{1}{m_{C}}\right)+\frac{k_{P}}{m_{C}}}, \quad \zeta=\frac{1}{2 \omega_{n}}\left[c\left(\frac{1}{m_{T}}+\frac{1}{m_{C}}\right)+\frac{k_{D}}{m_{C}}\right]
$$

To avoid thrust saturation, the proportional gain, $k_{P}$, is set arbitrarily to 500 , which does not change the openloop natural frequency significantly, due to the relatively large chaser's mass. Substituting the parameters for Kevlar tether from Table 1, yields $\omega_{n}=1.288 \mathrm{rad} / \mathrm{s}$. Choosing the stiffest tether parameters, ensures the greatest natural 
frequency, which in turn results in the greatest derivative gain, $k_{D}$, necessary to obtain the same level of a damping ratio, $\zeta$. The derivative gain, $k_{D}$, in Eq. (27) is chosen to obtain a non-oscillatory response, $\zeta=0.7$. Substituting $c=0$, yields $k_{D}=4508$. Integral term in Eq. (15) is set to $k_{I}=10$. Relatively small value does not change the system's dynamics and ensures cancelling the steady-state error.

Proportional and derivative gains in the chaser's attitude controller in Eq. (21) are obtained using linearized closed-loop attitude dynamics equations [21]. The natural frequency and damping ratio of the system are given by:

$$
\omega_{n}=\sqrt{\frac{K_{j}}{4 I_{j}}}, \quad \zeta=\frac{P_{j}}{\sqrt{K_{j} I_{j}}}
$$

where the subscript $j$ corresponds to a given body axis, i.e. $\mathrm{X}, \mathrm{Y}$ or $\mathrm{Z}$. The natural frequency of the system should not be close to the natural frequency of the translation motion, to avoid the resonances. Hence, the natural frequency of the closed-loop system for each body axis was chosen to be equal to $0.01 \mathrm{~Hz}$, which corresponds to $\omega_{n}=0.063$ $\mathrm{rad} / \mathrm{s}$. The damping constant for each body axis is set to 1. Substituting the elements of the inertia tensor given in Eq. (22), yields:

$$
\begin{aligned}
& \boldsymbol{K}=\operatorname{diag}[47,158,158] \\
& \boldsymbol{P}=\operatorname{diag}[375,1257,1257]
\end{aligned}
$$

The integral term in Eq. (21) is set to $\boldsymbol{K}_{I}=\operatorname{diag}[1,2,2] \cdot 10^{-3}$. Relatively small value does not change the system's dynamics and ensures cancelling the steady-state error.

\section{Target Alignment Angle and Angular Rate Analysis}

Six simulations for each tether model from Table 1 are conducted for a duration of 2 orbits which corresponds to approximately 200 minutes. The emphasis is put on analyzing the target attitude motion and the tether tension. Figure 4 presents the target alignment angle, as defined in Fig. 3, for all six tether models. The commanded torque in all cases is less than $50 \mathrm{Nm}$ and the chaser's attitude is stabilized with a $0.5^{\circ}$ accuracy and is not presented here. However, for the case with the elastic tether made of Nylon, the relative distance controller commands a force of the order of $1000 \mathrm{~N}$ for approximately $10 \mathrm{~s}$ just after the deorbit burn, in order to stabilize the system. This makes a typical reaction control system unfeasible for these cases and requires a system with greater thrust. For the tether 
made of Technora and Kevlar, the commanded force is less than $100 \mathrm{~N}$. In all cases, the system oscillates around the nadir vector as described in [10]. 




a) Nylon-LD

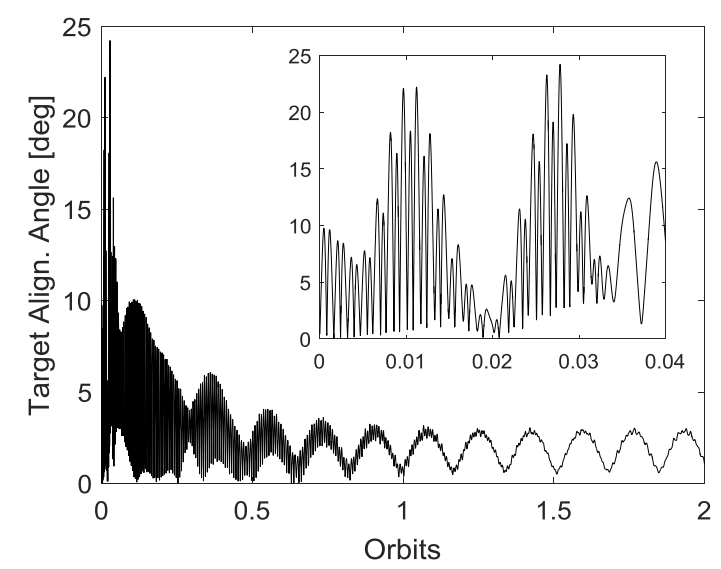

c) Technora-LD

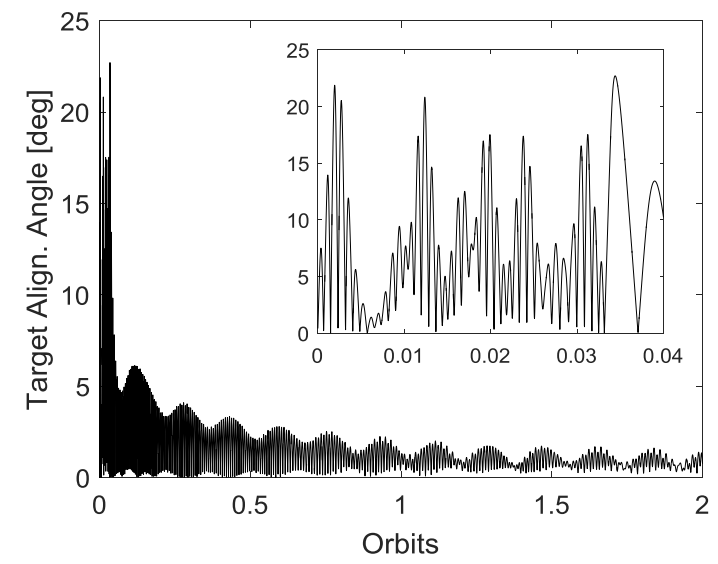

e) Kevlar-LD

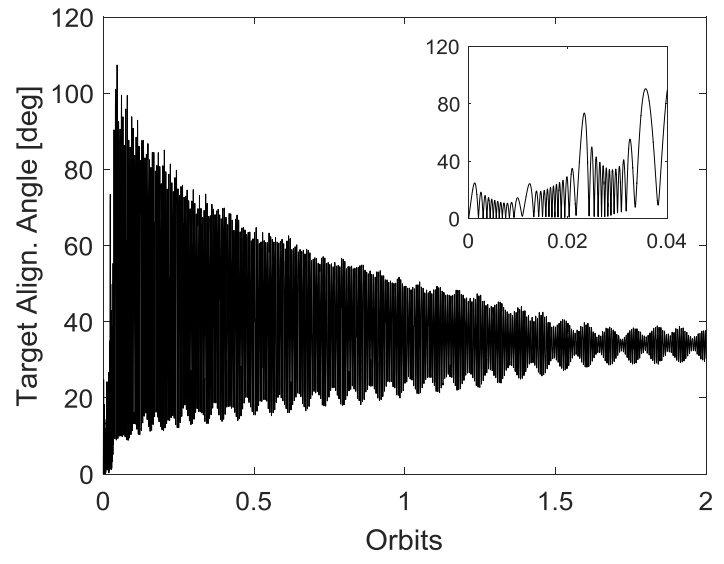

b) Nylon-HD

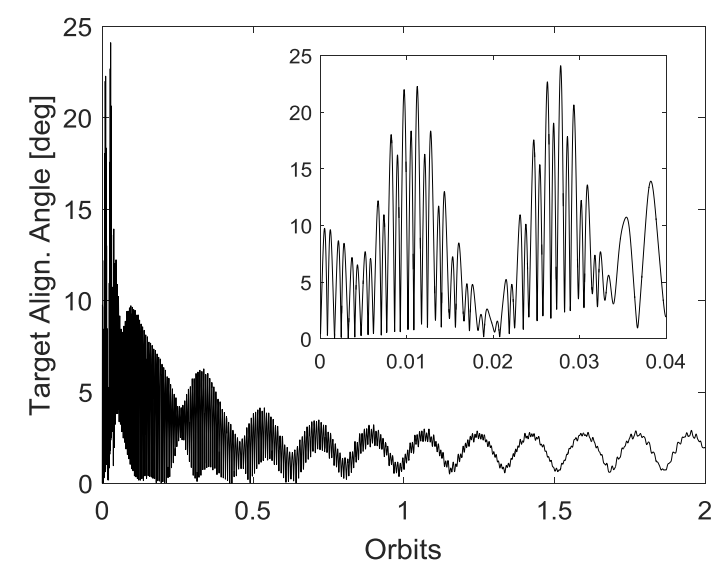

d) Technora-HD

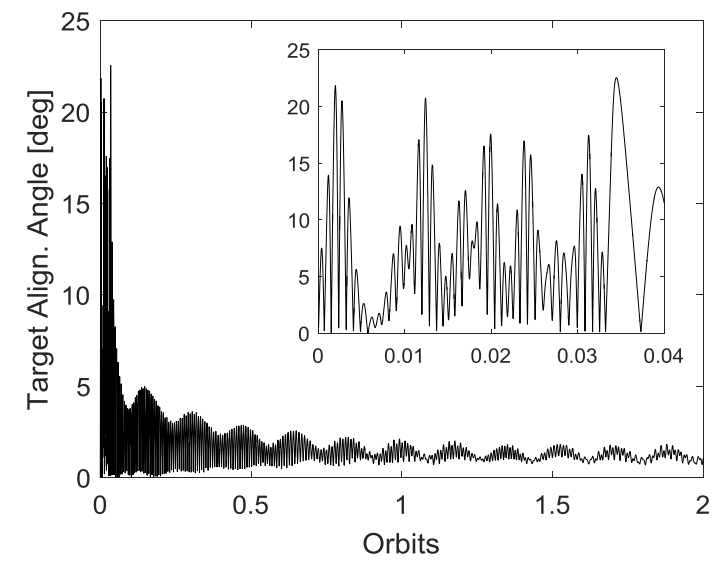

f) Kevlar-HD

Fig. 4 Target alignment angle for six tether models. Zoom from $t=0 \mathrm{~s}$ to $t=242 \mathrm{~s}$. A duration of the de-orbit burn is $200 \mathrm{~s}$, which corresponds to 0.033 orbit. 
Figures 4.a and 4.b show that for the elastic tethers, the maximum alignment angle reaches $110^{\circ}$, which is unacceptable in a realistic ADR mission scenario. An alignment angle greater than $90^{\circ}$, can lead to tether tangling and its eventual rupture, thus creating more debris/fragmentation. Furthermore, the system's mathematical model presented in Sec. II does not account for the accurate behavior when the alignment angle of either end body is greater than $90^{\circ}$. In this case, the tether starts to wrap up around the body, which requires refinements in the mathematical model.

The Nylon tether slowly damps the target attitude motion, but the target does not stabilize at the desirable alignment angle (equal 0), see Fig. 4.a and 4.b. Moderately stiff tethers made of e.g. Technora, significantly increase the damping of the end body attitude motion, which can be observed in Fig. 4.c and 4.d. The maximum alignment angle in both cases is lower than $25^{\circ}$ which means a very safe operation can take place. For the tether made of Technora, however, the alignment angle does not stabilize at the desirable alignment angle equal 0, but exhibits nondecaying oscillations with the amplitude equal to approximately $1^{\circ}$, see Fig. 4.c and 4.d.

Stiff tethers made of e.g. Kevlar have similar influence on target attitude motion than the moderately stiff tether made of Technora. This can be seen by comparing Fig. 4.e and 4.c. Stiffer tethers, however, damp the target's attitude motion more rapidly which can be seen shortly after the deorbit burn. The amplitude of the target alignment angle oscillations for the tether made of Kevlar is lower than for the tether made of Technora.

No significant difference in the attitude of the target is observed for the tethers with higher damping constant. This can be observed by comparing Fig. 4.a, 4.c. and 4.e with Fig. 4.b, 4.d and 4.f, respectively.

Time histories of the target's angular rate norm are presented in Fig. 5 for six tether models from Table 1. 


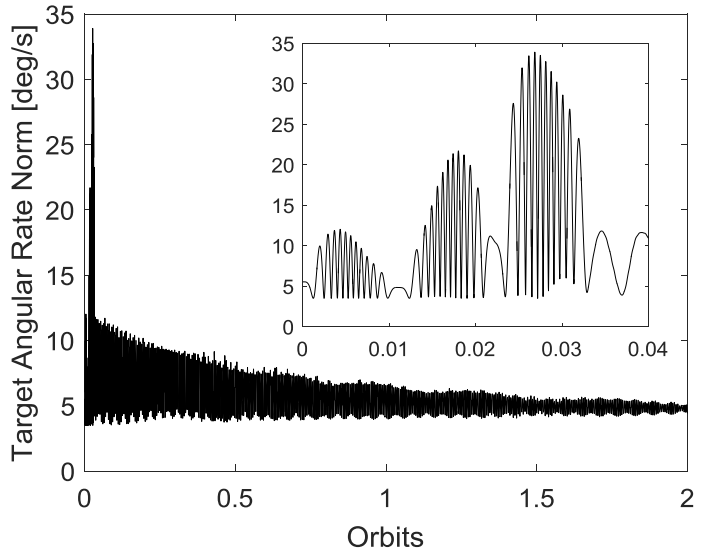

a) Nylon-LD

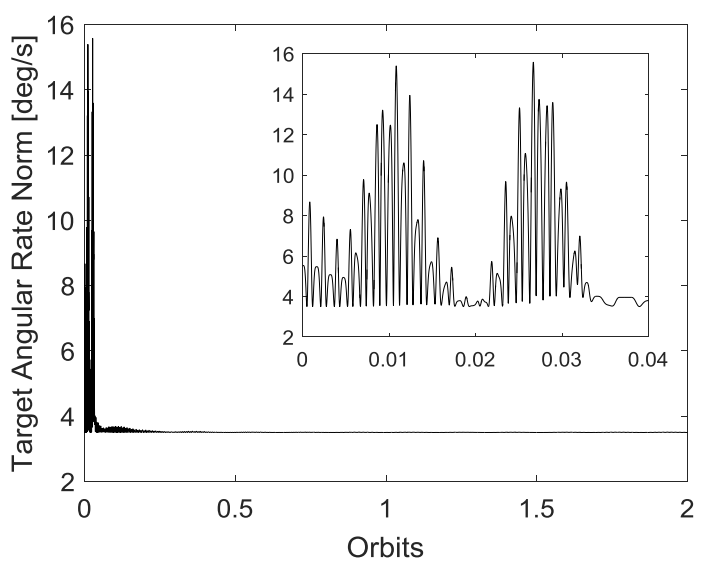

c) Technora-LD

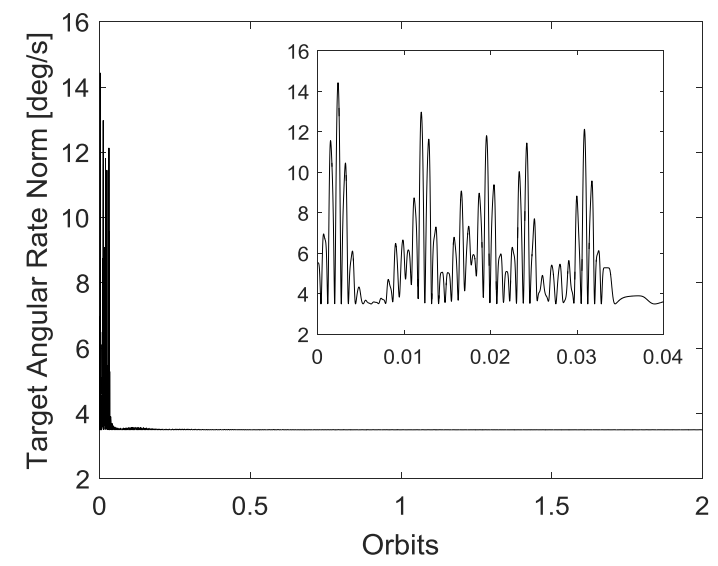

e) Kevlar-LD

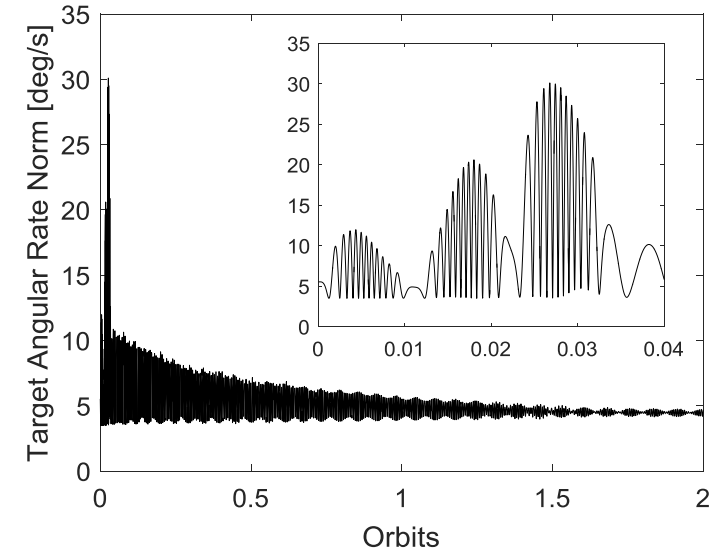

b) Nylon-HD

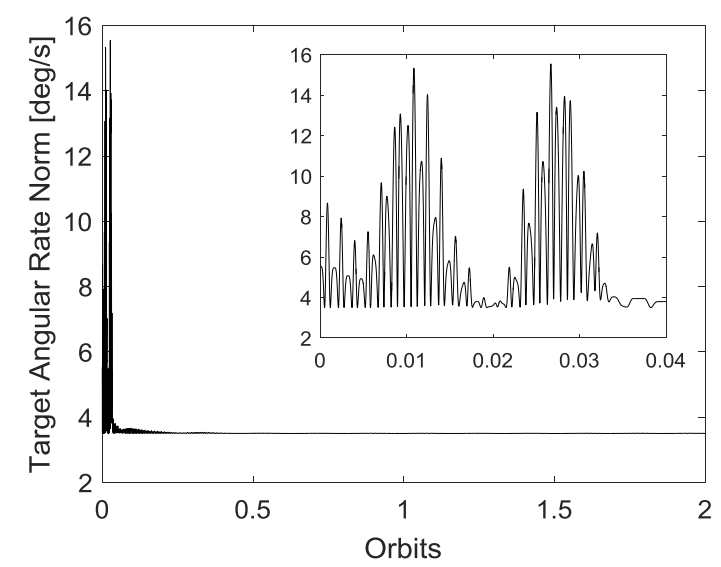

d) Technora-HD

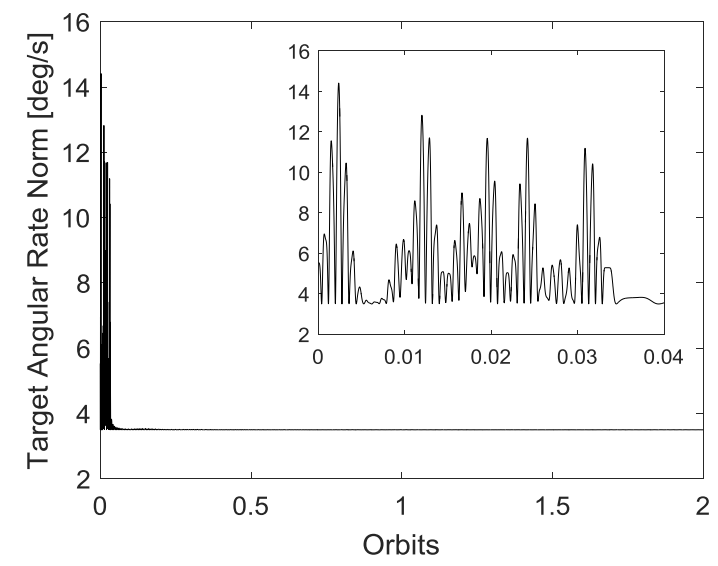

f) Kevlar-HD

Fig. 5 Target angular rate norm for six tether models. Zoom from $t=0 \mathrm{~s}$ to $t=242 \mathrm{~s}$. A duration of the de-orbit burn is 200 s, which corresponds to 0.033 orbit. 
Analyzing Fig. 5, similar conclusions can be made to those relating to the target alignment angle. Elastic tethers made of Nylon exhibit a higher angular rate during the deorbit burn and smaller damping of the angular rate, following the deorbit burn, than for the tethers made of Technora and Kevlar. For stiffer tethers, the angular rate oscillations during the deorbit burn are damped rapidly and stabilize at approximately $3.5 \%$ s. This value corresponds to the target's initial angular rate around the body $\mathrm{X}$ axis. Since for the stiffer tethers, the alignment angle after the deorbit burn is less than $10^{\circ}$, the tether tension damps the target's angular rate only around the body $\mathrm{Y}$ and $\mathrm{Z}$ axes. Hence, in the final configuration, the target spins around the body $\mathrm{X}$ axis which is almost aligned with the tether. Since the tether torsion is not included in the attitude-tether model, the rotation around the body $\mathrm{X}$ axis is not damped which is clearly observed in Fig. 5.c-f.

Similarly to the alignment angle analysis, no significant difference in the behavior of the target is observed for the tethers with higher damping constant. This can be observed by comparing Fig. 5.a, 5.c. and 5.e with Fig. 5.b, 5.d and 5.f, respectively.

The target attitude motion analysis for six tether models is summarized in Table 2. Maximum alignment angles and angular rate norms, in Table 2, are calculated for the full simulated scenario. Mean values are obtained for the time duration of the last 0.2 orbit, which corresponds to the last $1210 \mathrm{~s}$ of the simulation. Amplitudes have been defined as follows:

$$
\Delta x=\frac{x_{\max }-x_{\min }}{2},
$$

where maximum and minimum values are computed for the time duration of the last 0.2 orbit, similarly to the mean values.

Table 2 Target attitude motion analysis

\begin{tabular}{lcccccc}
\hline & \multicolumn{3}{c}{ Alignment Angle, deg } & \multicolumn{3}{c}{ Angular Rate Norm, deg/s } \\
\cline { 2 - 7 } Model & Max Value & Mean & Amplitude & Max Value & Mean & Amplitude \\
\hline Nylon-LD & 109.65 & 37.54 & 9.73 & 33.91 & 4.85 & 0.630 \\
Technora-LD & 24.23 & 1.91 & 1.21 & 15.57 & 3.50 & 0.006 \\
Kevlar-LD & 22.71 & 0.85 & 0.69 & 14.43 & 3.50 & 0.001 \\
Nylon-HD & 107.42 & 34.07 & 5.04 & 30.10 & 4.47 & 0.319 \\
Technora-HD & 24.11 & 1.89 & 1.09 & 15.54 & 3.50 & 0.004 \\
Kevlar-HD & 22.55 & 1.16 & 0.57 & 14.41 & 3.50 & 0.001 \\
\hline \hline
\end{tabular}

It can be seen, that the stiff tethers outperform the soft tethers, due to the significantly lower maximum alignment angle, which improves the safety of the ADR-towing mission and reduces the risk of tether tangling and 
rupture. For the stiff tethers, the alignment angle stabilizes approximately at the desirable $0^{\circ}$ attitude angle, with a $2^{\circ}$ accuracy which is acceptable in realistic ADR mission scenario. Furthermore, the stiff tethers damp the target angular rate around the transverse, $\mathrm{Y}$ and $\mathrm{Z}$ body axes.

\section{E. Tether Tension Analysis}

The tether tension for six tether parameters is presented in Fig. 6. Since the tether consists of 2 lumped masses and 3 tether links, the tension varies across the tether length. The tether tension presented in Fig. 6, however, corresponds to the tension assuming a single tether element, and therefore a massless tether model. In this simplified model, the tether is spanned between the tether attachment points. The tether tension is calculated as follows:

$$
\boldsymbol{T}=\xi\left[k\left(|\boldsymbol{l}|-l_{0}\right)+\left(c \frac{\mathrm{d}}{\mathrm{d} t}|\boldsymbol{l}|\right)\right] \hat{\boldsymbol{e}}
$$

where the tether length, $l_{0}$, equals $1000 \mathrm{~m}$, the spring and damping constants are given for the whole tether, and the tether unit vector, $\hat{\boldsymbol{e}}$, and the length vector, $\boldsymbol{l}$, are given by:

$$
\hat{\boldsymbol{e}}=\frac{\boldsymbol{l}}{|\boldsymbol{l}|}=\frac{\boldsymbol{R}_{T}+\mathbf{A}_{T}^{I} \boldsymbol{r}_{p o r t, T}-\boldsymbol{R}_{C}-\mathbf{A}_{C}^{I} \boldsymbol{r}_{p o r t, C}}{\left|\boldsymbol{R}_{T}+\mathbf{A}_{T}^{I} \boldsymbol{r}_{p o r t, T}-\boldsymbol{R}_{C}-\mathbf{A}_{C}^{I} \boldsymbol{r}_{p o r t, C}\right|}
$$

Finally, the time derivative of the tether length vector can be obtained as follows:

$$
\frac{\mathrm{d}}{\mathrm{d} t}|\boldsymbol{l}|=\left(\boldsymbol{V}_{p o r t, T}-\boldsymbol{V}_{p o r t, C}\right)^{T} \hat{\boldsymbol{e}}
$$

where the tether attachment points velocities are given by Eq. (11). Note that the tether tension in Eq. (30) is a vector expressed in the ECI frame. In Fig. 6, the norm of this vector is shown.

During the deorbits burn, the tether tension oscillates with the frequency depending on the system's parameters, such as the tether Young's modulus. The tension of the Nylon tether exhibits the oscillations with the lowest frequency, due to its lowest spring constant.

In the final configuration, the tether tension is stabilized at approximately constant value, which is $5 \mathrm{~N}$ for Kevlar and Technora tethers and $10 \mathrm{~N}$ for the tethers made of Nylon. The difference can be explained by the lower damping of the target attitude motion by the elastic tethers. As can be seen in Fig. 5.a and 5.b, the target is not stabilized at $0^{\circ}$ alignment angle configuration, but rather is rotated by approximately $40^{\circ}$. Due to the non-zero 
angular rate around the target's $\mathrm{X}$ axis, the target's precession requires to apply an additional torque by a tether force. This corresponds to a higher tether tension in the final configuration in Fig. 6.a and 6.b.

Similarly to the previous analysis, no significant difference in the behavior of the tether is observed for the tethers with higher damping constant. This can be observed by comparing Fig. 6.a, 6.c. and 6.e with Fig. 6.b, 6.d and 6.f, respectively. 




a) Nylon-LD

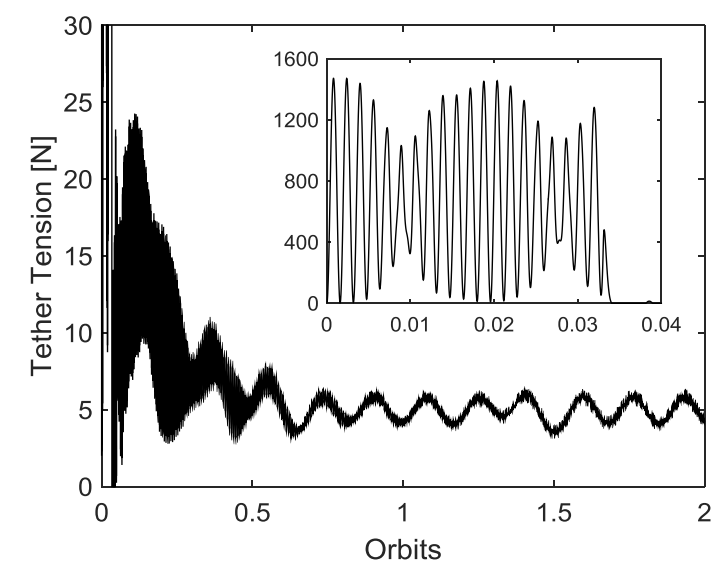

c) Technora-LD

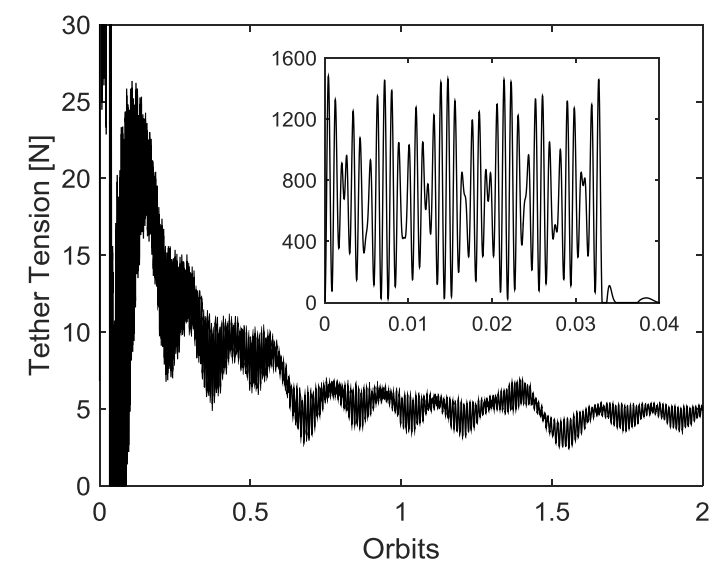

e) Kevlar-LD

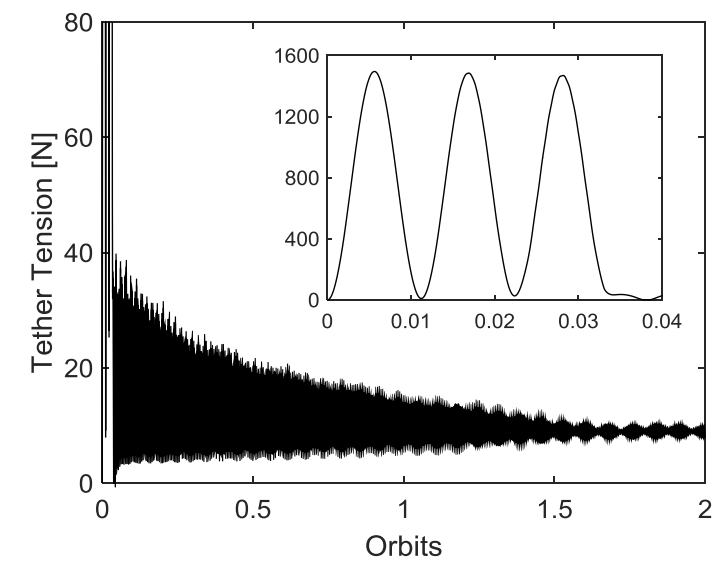

b) Nylon-HD

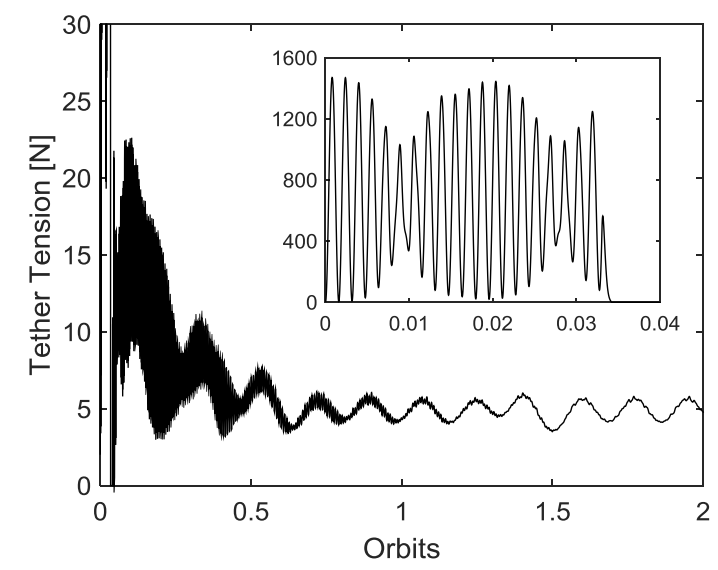

d) Technora-HD



f) Kevlar-HD

Fig. 6 Tether tension for six tether models. Zoom from $t=0 \mathrm{~s}$ to $t=242 \mathrm{~s}$. A duration of the de-orbit burn is 200s, which corresponds to 0.033 orbit. 
As it has been already explained, stiff tethers made of Technora and Kevlar result in lower tether tension in the final configuration. This corresponds to lower fuel consumption, as lower thrust is applied by the active chaser. Also note that when the feedback controller based on the tether tension or its elongation is used with the elastic tethers, several engineering challenges arise. Elastic tethers require less force for the same amount of elongation, hence the necessary thrust accuracy is greater. On the other hand, for a given tether tension, the required elongation is greater. Therefore, to keep the desired tension in the tether, the chaser needs to travel greater distances, see also $\Delta l$ column in Table 1. This causes the delays in the control system and hence larger control errors for elastic tethers which result in greater fuel consumption. For all these reasons, when the target attitude, safe operation and low fuel consumption are of primary concern, Kevlar is the optimal tether material for ADR-towing missions.

\section{Conclusion}

In this paper, the attitude motion of debris during tethered towing is investigated. The mathematical model consists of two end bodies modeled as rigid bodies with 6 degrees of freedom and the tether is discretized into 2 point masses and 3 tether links, each modeled as a spring-damper system. A simple guidance and control system is added to keep a small residual tension in the tether, following the deorbit burn. It is assumed that the magnitude of the target initial angular rate vector is $5.5 \%$, as expected in a realistic ADR mission scenario. Six tether models are investigated, and the influence of the tether elasticity and damping constant is analyzed.

The tether parameters have significant influence on the attitude motion of the target. Careful tether design reduces the risk of the target rotation excitation even when the step deorbit burn is performed. The developed methodology can be applied to select the optimum tether material for ADR towing missions. Small initial angular rates of the target, prior to the deorbit burn, can be tolerated. The numerical simulations show that the proposed control technique can keep the target alignment angle less than $25^{\circ}$ for more stiff tethers which is due to the increased damping of the target attitude motion caused by the restoring torque generated by the tether tension, thus improving safe towing substantially. The study reveals that for safety reasons, elastic tethers should be avoided, because they can lead to target attitude motion excitation and thus debris collision and fragmentation. It is shown, that the tether damping constant has no significant influence on the target rotation. Furthermore, elastic tethers are found to be more difficult to control, due to less tether tension required to maintain the same tether elongation. For stiff tethers, a relatively simple guidance and control system is shown to be sufficient to keep the tether tension of 
the order of $4 \mathrm{~N}$, to stabilize the tether and target dynamics, in a towing mission. The findings of this paper may be useful not only for the stabilization of the system during the coasting phase of active space debris removal missions, but also in the follow-up of the deorbit burn and stabilization phase, just after net capture of the debris target.

\section{References}

[1] Beletsky, V. V., and Levin, E. M., Dynamics of Space Tether Systems, Advances in the Astronautical Sciences, Vol. 83, American Astronautical Society Publications, San Diego, CA, 1993, pp. 20-33.

[2] Kessler, D. J., Johnson, N. L., Liou, J. C., and Matney, M., “The Kessler Syndrome: Implications to Future Space Operations," 33rd Annual AAS Guidance and Control Conference, AAS Paper 2010-016, Breckenridge, CO, Feb. 2010.

[3] Liou, J. C., “An Active Debris Removal Parametric Study for LEO Environment Remediation,” Advances in Space Research, Vol. 47, No. 11, 2011, pp. 1865-1876.

doi: 10.1016/j.asr.2011.02.003

[4] Reed, J., and Barraclough, S., "Development of Harpoon System for Capturing Space Debris," 6th European Conference on Space Debris, ESA SP-723, Darmstadt, Germany, April 22 - 25, 2013.

[5] Retat, I., Bischof, B., Starke, J., Froth, W., and Bennell, K., "Net Capture System,” 2nd European Workshop on Active Debris Removal, Paper No. 4.3, Quentin, Paris, June 18 - 192012.

[6] Reed, J., Busquets, J., and White, C., “Grappling System for Capturing Heavy Space Debris,” 2nd European Workshop on Active Debris Removal, Paper No. 4.2, Quentin, Paris, June 18 - 19, 2012.

[7] Nishida, S., and Yoshikawa, T., "Space Debris Capture by a Joint Compliance Controlled Robot," Proceedings of the 2003 IEEE/ASME International Conference on Advanced Intelligent Mechatronics (AIM 2003), Vol. 1, Kobe, Japan, July 20 - 24, 2003, pp. 496-502. doi: 10.1109/AIM.2003.1225145

[8] Jasper, L., Schaub, H., Seubert, C., Valery, T., and Yutkin, E., “Tethered Tug for Large Low Earth Orbit Debris Removal,” AAS/AIAA Astrodynamics Specialists Conference, Paper No. AAS 12-252, Charleston, SC, January 29 - February 2, 2012.

[9] Jasper, L., and Schaub, H., "Input Shaped Large Thrust Maneuver with a Tethered Debris Object," Acta Astronautica, Vol. 96, March-April 2014, pp. 128-137.

doi: 10.1016/j.actaastro.2013.11.005

[10] Jasper, L., and Schaub, H., "Tether Design Considerations for Large Thrust Debris De-Orbit Burns," AAS/AIAA Space Flight Mechanics Meeting, Paper No. AAS 14-443, Santa Fe, NM, January 26 - 30, 2014.

[11] Jasper, L., and Schaub, H., "Tethered Towing Using Open-Loop Input-Shaping and Discrete Thrust Levels," Acta Astronautica, Vol. 105, No. 1, Dec. 2014, pp. 373-384. 
doi: 10.1016/j.actaastro.2014.10.001

[12] Jasper, L., “Open-Loop Thrust Profile Development for Tethered Towing of Large Space Objects,” Ph.D. Thesis, Aerospace Engineering Sciences Dept., Univ. of Colorado, Boulder, CO, 2014.

[13] Linskens, H. T. K., and Mooij, E., "Tether Dynamics Analysis and Guidance and Control Design for Active Space-Debris Removal," Journal of Guidance, Control and Dynamics, Vol. 39, No. 6, 2016, pp. 1232-1243. doi: 10.2514/1.G001651

[14] Aslanov, V. S., and Yudintsev, V. V., "Dynamics of Large Space Debris Connected to Space Tug by a Tether,” Journal of Guidance, Control and Dynamics, Vol. 36, No. 6, 2013, pp. 1654-1660. doi: $10.2514 / 1.60976$

[15] Aslanov, V. S., and Yudintsev, V. V., "Dynamics of Large Space Debris Removal Using Tethered Space Tug," Acta Astronautica, Vol. 91, Oct.-Nov. 2013, pp. 149-156. doi: 10.1016/j.actaastro.2013.05.020

[16] Aslanov, V. S., and Ledkov, A. S., "Dynamics of Towed Large Space Debris Taking Into Account Atmospheric Disturbance,” Acta Mechanica, Vol. 225, No. 9, 2014, pp. 2685-2697. doi: 10.1007/s00707-014-1094-4.

[17] Biesbroek, R., Hüsing, J., and Wolahan, A., "System and Concurrent Engineering for the eDeorbit Mission Assessment Studies," 6th International Systems and Concurrent Engineering for Space Applications Conference, Stuttgart, Germany, Oct. 2014.

[18] Gómez, N. O., and Walker, S. J. I., "Earth's Gravity Gradient and Eddy Currents Effects on the Rotational Dynamics of Space Debris Objects: Envisat Case Study,” Advances in Space Research, Vol. 56, No. 3, August 2015, pp. 494-508. doi: 10.1016/j.asr.2014.12.031

[19] Kucharski, D., Kirchner, G., Koidl, F., Fan, C., Carman, R., Moore, C., et al., "Attitude and Spin Period of Space Debris Envisat Measured by Satellite Laser Ranging," IEEE Transactions on Geoscience and Remote Sensing, Vol. 52, No. 12, Dec. 2014, pp. 7651-7657. doi: 10.1109/TGRS.2014.2316138

[20] Perez, E., SOYUZ from the Guiana Space Centre User's Manual, 1st ed., Arianespace, Evry-Courcouronnes Cedex, France, June 2016.

[21] Schaub, H., and Junkins, J. L., Analytical Mechanics of Aerospace Systems, AIAA Education Series, AIAA, Reston, VA, 2003, pp. 381-396.

[22] Markley, F. L., and Crassidis, J. L., Fundamentals of Spacecraft Attitude Determination and Control, Space Technology Library, Springer, New York, 2014, pp. 289-302. 
doi: $10.1007 / 978-1-4939-0802-8$

[23] Li, G., Zhu, Z. H., Cain, J., Newland, F., and Czekanski, A., "Libration Control of Bare Electrodynamic Tethers Considering Elastic-Thermal-Electrical Coupling," Journal of Guidance, Control and Dynamics, Vol. 39, No. 3, 2016, pp. $642-654$. doi: $10.2514 / 1 . G 001338$

[24] Houten, M., "Invitation to Tender AO/1-7477/13/ML/MH: Advanced Guidance, Navigation and Control for Active Debris Removal,” 13.1EC.04, ESA ESTEC, Noordwijk, The Netherlands, 2013

[25] Li, G. Q., and Zhu, Z. H., "Long-term dynamic modeling of tethered spacecraft using nodal position finite element method and symplectic integration," Celestial Mechanics and Dynamical Astronomy, Vol. 123, Issue 4, 2015, pp. 363-386. doi: $10.1007 / \mathrm{s} 10569-015-9640-5$ 
2017-02-22

\section{Debris rotation analysis during tethered towing for active debris removal}

Jaworski, P.

American Institute of Aeronautics and Astronautics

Jaworski P, Lappas V, Tsourdos A, Gray I, Schaub H, Debris rotation analysis during tethered towing for active debris removal, Journal of Guidance, Control, and Dynamics, Vol. 40, No. 7 (2017), pp1769-1778

http://dx.doi.org/10.2514/1.G002390

Downloaded from Cranfield Library Services E-Repository 